\title{
Photography and imagery
}

\section{This week's product review covers some of the latest advances in photomicrography and processing of micrograph images.}

The rapid developments in imaging technology have led to greater demands on the recording materials available. To meet these demands, Kodak has introduced ' $T$ Grain' emulsions, incorporating carefully oriented flat silver halide grains that allow much faster emulsions, without the usual trade-off in graininess. This is incorporated in Kodak's latest colour negative films, and will soon appear in X-ray films also. Kodak's range of materials for scientific use includes papers for recording oscilloscope traces and plates for electron microscopy, photomicrography, holography, spectroscopy and astronomy. High resolution plates, for example, are capable of recording over 2,000 lines per $\mathrm{mm}$. Kodak Technical Pan film 2415 ('EsterAH' Base) is particularly useful, allowing enlargements of $\times 25$ and more with little perceptible grain. Also from Kodak, the Sp2000 motion analysis system achieves video recording of high speed events at between 60 and 2,000 frames per second. The frame may also be divided into up to 6 horizontal segments, providing 12,000 frames per second.

Circle No. 100 on Reader Service Card.

Bausch \& Lomb has published two new brochures that describe their video-based image analysers. Common applications of image analysis are: bacterial colony counts, airborne contaminant analysis, fibre measurement in the textile industry, aerial and satellite photograph analysis and structural integrity analysis of building materials.

Circle No.101 on Reader Service Card.

- Dapple System, Inc. has published an image analysis brochure describing the Image-Plus + system for automatic measurement of features in video images. The various methods for producing the image signal, from an optical microscope, SEM, or TEM, are detailed and stereological analysis techniques discussed. The descriptions include the method of acquisiton and storage in the computer memory of the grey-scale image and the image array processing functions available to enhance this image before measurement. Data processing and statistical analysis routines required for quantitative stereology are described and illustrated by example.

Circle No. 102 Reader Service Card.

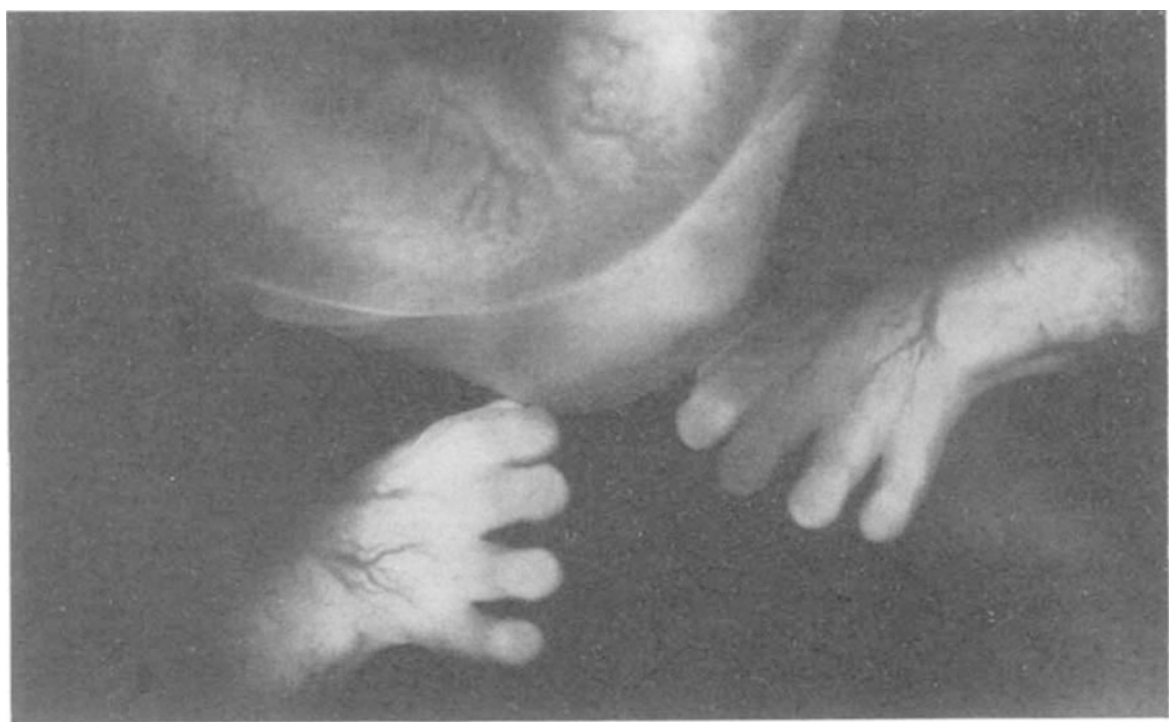

A human embryo after 12 weeks of differentiation, photographed by Dr R. Jonas. The instrument used was the Wild Heerbrugg M 400 Photomakroskop. The $M \mathbf{4 0 0}$ has been specially developed for 'macroscopy', that is, for the magnification range of $1: 1$ to $20: 1$, where the close-up range of an ordinary camera is no longer adequate, but where the visual field of the microscope becomes too small. The photographic range of the $M \mathbf{4 0 0}$ is in fact from $1: 1$ to $60: 1$, and there is a macrozoom objective (1:5) to provide 'stepless' changes in magnification. Further features of the $P 400$ include binocular observation, working distances of $\mathbf{4 2 - 1 8 8} \mathrm{mm}$ and an automatic system to determine exposure. Circle No. 103 for further details.

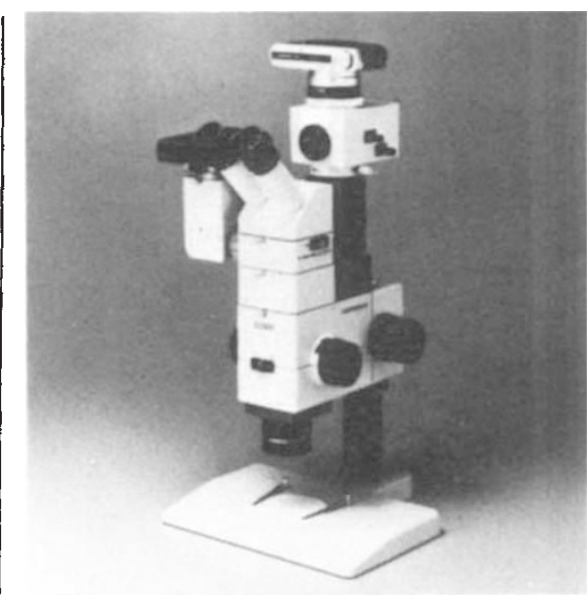

The $\mathrm{SZH}$ in a configuration for photomicrography.

- The building-block design of the new Olympus SZH zoom stereo microscope system makes it especially suited for photomicrography. As well as the PM Series photomicrographic system, Olympus OM Series SLR cameras can be fitted. The binocular viewing tube, inclined at $45^{\circ}$, is designed to permit easy focusing for photomicrography, and the distortion-free objective lenses, in magnifications of 0.5 , $0.75,1,1.5$ and 2 , provide clear images without the need for auxiliary lenses. Eyepieces are available with four different magnifications, 10, 15, 20 and $30 \times$. Circle No. 104 on Reader Service Card.

Photonics Microscopy, Inc. of Illinois has introduced the Model C1966, a highspeed video image processing system designed for real time applications. The C1966 features analog video enhancement and digital image processing through frame memories that permit subtraction, averaging, filtering and other image processing functions. These techniques make it possible to view microscopic structures and events that cannot be seen in the optical image. The $\mathrm{C} 1966$ can be used with video cameras and X-ray through infrared wavelength sources can be accommodated through the selection of specific cameras. Three frame memories of 640 by 438 lines permit extensive image manipulation in real time on the video monitor, while the image may also be recorded permanently on videotape or videomicrograph. The C1966. will interface with most popular computers. Circle No.105 on Reader Service Card.

These notes are based on information provided by the manufacturers. For further details circle the appropriate numbers on the Reader Service Card bound inside the journal. 
The 3090 pulsed light fibre-optic sensor from Oriel Scientific is designed to make a measurement of flourescence, absorption, reflection or scattered light using fibre-optic probes called 'optrodes', and will make the measurement in moderate ambient light. A full range of glass, silica, and liquid light guides allows measurements to be made in radioactive areas, fermentation systems, high voltage or explosive areas, biological hazard areas and marine locations. The analyser has its own control logic and will interface to a microcomputer such as the PET or Apple II. Circle No. 106 on Reader Service Card. - Signals passed down optical fibres will not reach their destination if there is a break in the cable. Now that optical fibres are widely used in communications equipment, accurate methods of precisely locating breaks are extremely important. One such technique is optical time domain reflectometry (OTDR) which is capable of providing accurate information on faults in cables several metres in length. EG\&G Instruments have recently announced their Model 4400 Boxcar, a system that incorporates signal processor, boxcar averager and gated integrator in a single unit, for use in OTDR experiments. OTDR relies on measuring the backscatter of a light beam that is passed down a fibre optic cable. Since the signal power and backscatter levels are very low, sophisticated timing electronics and signal processors are needed.

Circle No. 107 on Reader Service Card.

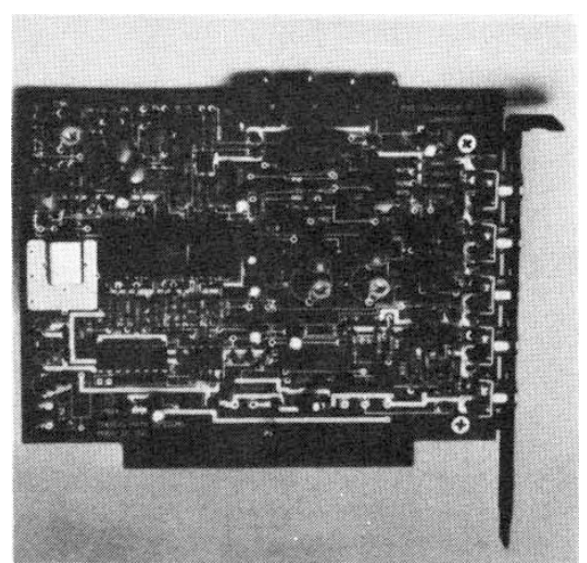

\section{The Colorverter from Chorus}

New from Chorus Data Systems, Inc., the Colorverter is a low-cost converter which changes NTSC composite colour signals into analog RGB. Colorverter may be used in conjunction with the Chorus PCEYE Video Capture System to digitize colour signals from colour video cameras, VCRs, and laser disks. The user has control of the colour intensity, tint (hue), brightness, and contrast. EIA RS-170A sync is available on all signals and there is a separate monochrome output for blackand-white applications. Applications include slide generation, inspection (where colour distinction is important) and medical/scientific classification. Systems and software support for many of these applications are available.

Circle No.108 on Reader Service Card.
- Several different imaging techniques are incorporated in the Kevex Microanalyst 8000 energy dispersive X-ray analyser for electron microscopists. Imagex is a sophisticated software package that allows the characteristic $X$ rays and other electron column signals to be digitized, stored and manipulated. Images are divided into a $1,024 \times 1,024$ pixel grid and each pixel can be stored as one of up to 64,000 levels. The Kevex Microanalyst 8000 is also able to display colour images. Different colours can be designated to different elements, thereby allowing accurate and easy to interpret displays, photographs or printer outputs to be produced.

Circle No. 109 on Reader Service Card.

- The Model $\mathbf{8 5 0}$ is a new image analysis system for biomedical research from Spatial Data Systems. It is a complete turnkey system for statistics database management and image capture, enhancement, segmentation, analysis, storage and retrieval. The hardware is configured to allow the operator to input images from film or microscope slides, to enhance them and gather statistics, and then to store on disk, display or print the results. The new system includes a camera, light table, alphanumeric terminal, colour monitor, joystick interface, printer, LSI II CPU, Winchester technology disk, eight-inch floppy disk, an EyeCom II real-time image processor, desk workstation and complete software. The system is available in a range of configurations.

Circle No.110 on Reader Service Card.

\section{Copies of articles from this publication are now available from the UMI Article Clearinghouse.}

\section{For more information ahout the \\ ('learinghouse, please fill out and mail back \\ the coupon helow: \\ (Xitiolinghouse.}

Yes! I would like to know more about UMI Article Clearinghcuse.

I am interested in electronic ordering through the following system(s):

$\square$ DLALOG/Dialorder

$\square$ ITT Dialcom

$\square$ OnTyme

$\square$ OCLC ILL Subsystem

$\square$ Other (please specify)

$\square$ I am interested in sending my order by mail.

$\square$ Please send me your current catalog and user instructions for the system(s) I checked above.

Name

Title

Institution/Company

Department

Address

City

State ip

Phone (

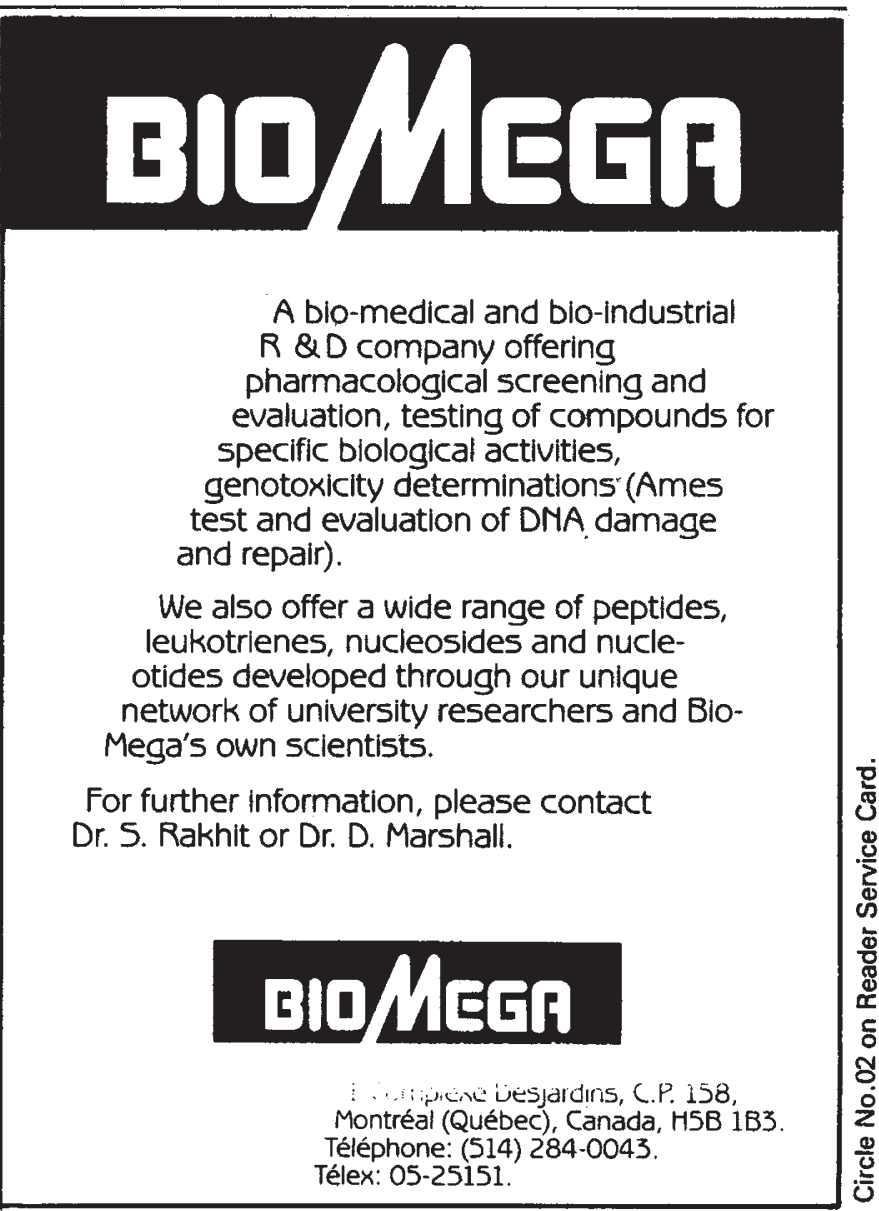

pharmacological screening and

evaluation, testing of compounds for specific biological activities, genotoxicity determinations (Ames test and evaluation of DMA damage and repair).

We also offer a wide range of peptides, leukotrienes, nucleosides and nucleotides developed through our unique network of university researchers and BioMega's own scientists.

For further information, please contact r. S. Rakhit or Dr. D. Marshall.
Télex: 05-25151. 